\title{
Rolling Element Bearing Condition Monitoring using Filtered Acoustic Emission
}

\author{
Sudarsan Sahoo, J. K. Das, Bapi Debnath
}

School of Electronics Engineering, KIIT University, India

\begin{tabular}{l}
\hline \hline Article Info \\
\hline Article history: \\
Received May 26, 2018 \\
Revised Jul 1, 2018 \\
Accepted Jul 12, 2018 \\
\hline
\end{tabular}

Keyword:

Acoustic emission

Active noise cancellation

Bearing fault

EDM

Wavelet transform

\begin{abstract}
The defect present in the bearing of a rolling element may affect the performance of the rotating machinery and may reduce its efficiency. For this reason the condition monitoring of a rolling element bearing is very essential. So many measuring parameters are there to diagnose the fault in a rolling element bearing. Acoustic signature monitoring is one of them. Every rolling element bearing has its own acoustic signature when it is in healthy condition and when the bearing get defected then there is a change in its original acoustic signature. This change in acoustic signature can be monitored and analyzed to detect the fault present in the bearing. But the noise present in the acquired acoustic signal may affect the analysis. So the noisy acoustic signal must be filtered before the analysis. In this work the experiment is performed in two stages. In first stage the filtration of the acquired acoustic signal is done by employing the active noise cancellation (ANC) filtering techniques. In second stage the filtered signal is used for the further analysis. For the analysis initially the static analysis is done and then the frequency and the time-frequency analysis is done to diagnose the defect in the bearing. From all the three analysis the information about the defect present in the bearing is well detected.
\end{abstract}

Copyright (C) 2018 Institute of Advanced Engineering and Science. All rights reserved.

Corresponding Author:

Sudarsan Sahoo,

School of Electronics Engineering,

KIIT University,

Bhubaneswar, India.

Email: sudarsan_iisc@yahoo.in

\section{INTRODUCTION}

The rolling element bearing has its own acoustic signature in its healthy condition. This acoustic signature gets changed when defects occur in the bearings. Proper analysis of this change in acoustic signature gives precise information about this defect. But the acoustic signature may get corrupted with the noise produced by other components. So this noise should be filtered before its analysis to diagnose the defect. The active noise control (ANC) is employed for the filtering of this noisy acoustic signal. The ANC is implemented by using the adaptive filters.

So many techniques can be found in literature to diagnose the defect in bearings. P.K. Kankar et al., shows the application of continuous wavelet transform to diagnose the fault in bearings [1], [2]. Wei Li et al., shows the fault analysis based on the spectrum images of the vibration signal [3]. In some past work application of wavelet can be found [4], [5]. It can be noticed that time-frequency analysis along with the static analysis of vibration signal can be used detect the fault in the bearing [6]-[8]. B. Li et al. shows the use of morphological filters for gear fault detection [9]. A. Albarbar et al., shows the acoustic monitoring of engine fuel injection [10]. Fault diagnosis for the multilevel inverter switch is found in literature [11]. M.A. Talib et al., shows the fault identification using depolarisation current analysis [12]. In addition to that adaptive filters are used to improve the SNR (signal to noise ration) of the signal before its analysis to diagnose the defect. C.W. Liao et al., shows the use of adaptive filter to improve the signal to noise ratio 
[13]. In literature it can be found the application of LMS algorithm in adaptive filters as the de-noising tool to cancel the noise [14], [15].

In this work initially the ANC is implemented on acquired acoustic signal. For ANC the de-noised principle of noise removal is followed. In the de-noising process three adaptive algorithms are tested and their performance is compared in terms of their SNR (signal to noise ratio) and MSE (mean square error). The LMS, wavelet and EDM algorithm is used for the same. As EMD algorithm is found as the best then the filtered acoustic signal from the EDM de-noising is used for the further processing and analysis. The analysis to detect the fault is done in time, frequency and Time-frequency domain. For this experiment one healthy bearing and three defective bearing is used.

\subsection{Active noise cancellation}

Active noise cancellation is used for the pre processing of the acoustic signal. Acoustic signals are always contaminated with noises and other external interferences, which must be removed or filtered before further processing and analysis.

\subsubsection{LMS de-noising}

The least mean square (LMS) algorithm is used as a de-noising tool for the ANC. It is used to update the adaptive filter coefficients as:

$$
w_{l}(n+1)=w_{l}(n)+\mu x(n-1) e(n)
$$

The ANC steps using the Least Mean Square algorithm is explained briefly as follows [11]:

a) The selection of the step size $\mu$ and the filter length $L$ is made.

b) The output of the adaptive filter is calculated as $y(n)=\sum_{l=0}^{L-1} w_{l}(n) x(n-1)$

c) The error signal is calculated as $e(n)=d(n)-y(n)$

d) The coefficients of the adaptive filter is updated by using the following formula :

$$
w_{l}(n+1)=w_{l}(n)+\mu x(n-l) e(n),
$$

where $l=0,1 \ldots ., L-1$.

The selection of the step size value is very important as it affects the convergence speed. The proper selection of filter length is also very important [12].

\subsubsection{Empirical mode decomposition}

This is another adaptive algorithm used for noise cancellation. This is suitable when the noise is non-stationary in nature. This algorithm is based on empirical basis functions. The original signal $x(t)$ is decomposed into the set $\left\{\mathrm{r}_{\mathrm{J}}, \mathrm{c}_{\mathrm{J}}, \mathrm{c}_{\mathrm{J}-1}, \ldots \ldots \ldots, \mathrm{c}_{1}\right\}$ where $\mathrm{c}_{\mathrm{j}}, \mathrm{j}=1, \ldots \ldots, \ldots \mathrm{J}$, represent intrinsic mode functions (IMF) and $\boldsymbol{r}_{\boldsymbol{J}}$ are residual terms.

$$
x(t)=\sum_{J=1}^{J} C_{J}(t)+r_{J}(t)
$$

The Empirical mode decomposition is an adaptive method to recognize oscillations from the signal $\boldsymbol{x}(\boldsymbol{t})$. Like discrete wavelet transfer, EMD method decompose a signal into so-called intrinsic mode functions(IMF).

\subsubsection{Wavelet de-noising}

This technique is one of the most popular and efficient techniques for the filtering of the noise. It starts with the decomposition of the signal into successive approximation and details. Wavelet de-noising performs correlation analysis. The expected value of the output turn out to be maximum if the input noisy signal looks a lot like the picked mother wavelet function. As the wavelet transform is linear it works best for the additive noise.

\subsection{Time domain study}

In time domain analysis the statistical features are computed from the vibration signature. By comparing these statistical features the faults in the system can be identified. The statistical parameters used 
for the time domain analysis are RMS, skewness, mean, peak value, crest factor, kurtosis, standard deviation, clearance factor, impulse factor and shape factor.

\subsection{Frequency domain study}

In time domain analysis some information may not be revealed so frequency domain can is used to reveal those information which is not possible in time domain. The signal in time domain is basically converted to frequency domain by employing the Fourier transform. In this analysis the acoustic signal peak is displayed in the frequency spectrum and provide the information in frequency domain. The characteristic fault frequencies can be calculated by the following equations:

$$
\begin{aligned}
& \text { ORF (outer race fault })=\frac{N}{2} \omega_{n}\left(1-\frac{d}{D} \cos \alpha\right) \\
& \operatorname{IRF}(\text { Inner race fault })=\frac{N}{2} \omega_{n}\left(1+\frac{d}{D} \cos \alpha\right)
\end{aligned}
$$

\subsection{Time-frequency study}

In some cases time or frequency analysis alone may not give adequate information about the fault in the rotating machine. So time-frequency analysis is needed for this purpose to give better analysis of the fault. Wavelet transform is used for this purpose. The signals are processed by the wavelet transform to generate the two dimensional map of WT coefficients to get the required time frequency information. It provides the information simultaneously both in time and scale. In time-frequency methods of fault detection the contour plots are visually observed. The fault can be detected by visually monitoring the changes that occurred in the features of the distribution in the contour plots.

\section{PERFORMANCE COMPARISON OF ANC FILTERING TECHNIQUES}

In this section the performance of the ANC filtering techniques used to remove the noise is compared. Comparison is made on the basis of SNR (signal to noise ratio) and MSE (mean square error). In the experimental setup one defective bearing is placed. Then the acoustic signal is acquired. Then the three ANC techniques are implemented on these acquired acoustic signals. The acquired noisy acoustic signal and the filtered signal after ANC is shown in Figure 1. The comparison parameters of the three de-noising ANC techniques are tabulated in Table 1.

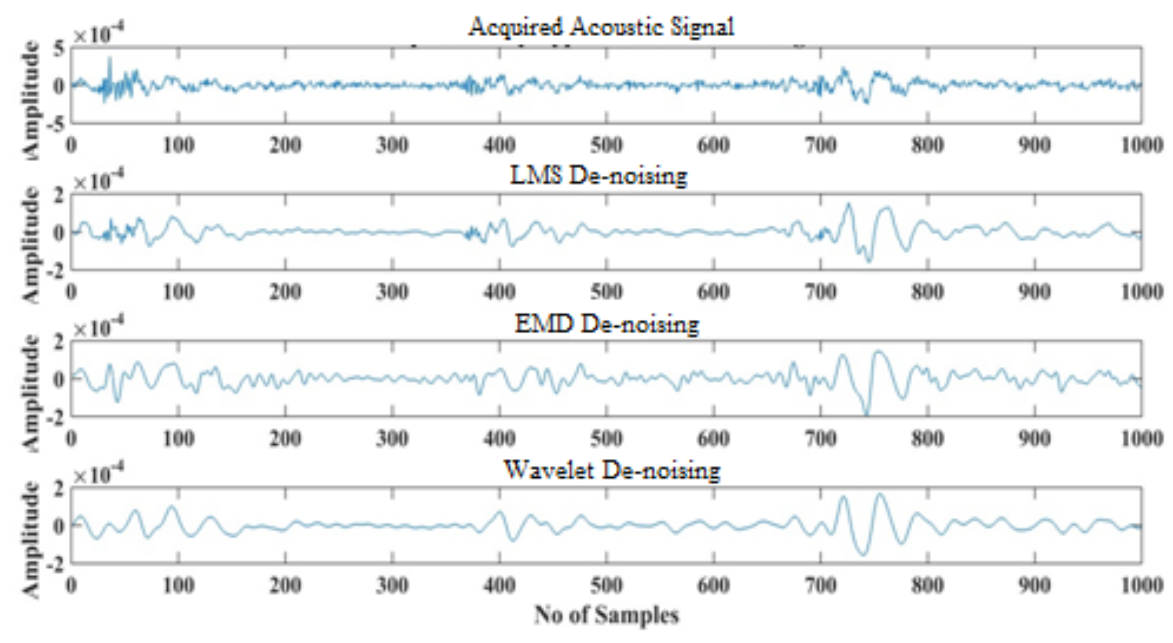

Figure 1. Comparison of ANC techniques

Table 1. Parameters Comparison for ANC Techniques

\begin{tabular}{ccc}
\hline ANC Techniques & SNR & MSE \\
\hline LMS & 11.068 & 0.0283 \\
EMD & 14.863 & 0.0210 \\
Wavelet & 13.061 & 0.0264 \\
\hline
\end{tabular}


From this comparison EMD is found better. So for the pre-processing stage the EMD active noise cancellation is selected and implemented on all the acoustic signals acquired from the experimental set-up and then the filtered acoustic signals are used for the further processing and analysis. The filtered acoustic signals are shown in Figure 5.

\section{EXPERIMENT}

To implement the proposed method of diagnose the defect present in the bearing an experimental set-up is made. The experimental set-up and its model is shown in Figure 2 and Figure 3 respectively. It consists of a single phase induction motor of $0.5 \mathrm{hp}$ and its speed is $1400 \mathrm{RPM}$ with no load at $230 \mathrm{~V}$ and $50 \mathrm{~Hz}$ supply.

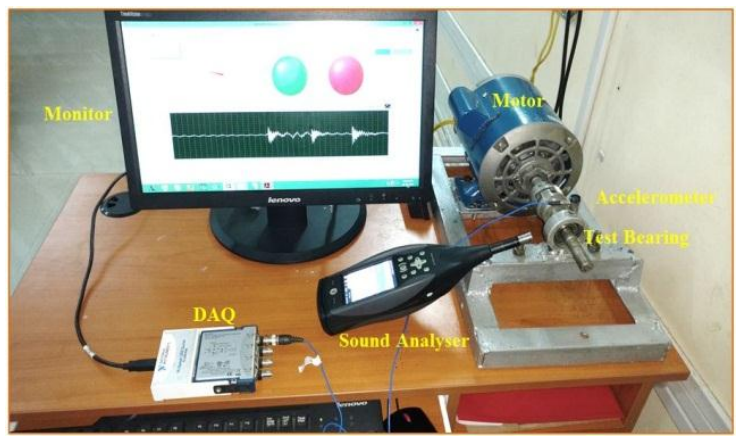

Figure 2. Hardware set-up for the experiment

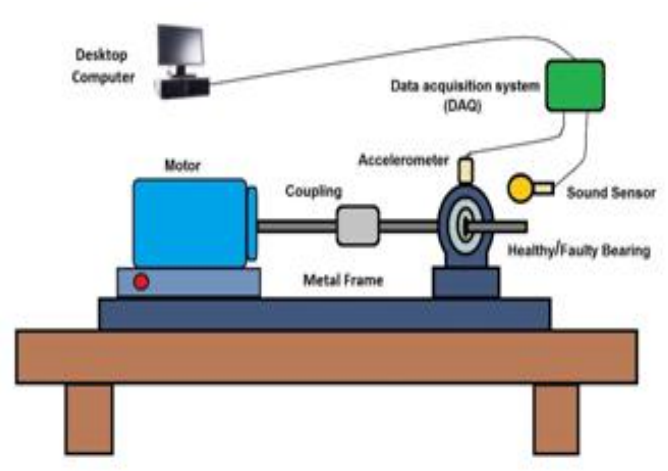

Figure 3. Model of set-up for the experiment

The acoustic data is acquired using a Brüel \& Kjær 2 channel handheld Sound analyser. The bearings under test are mounted on the shaft of the motor. For this work one healthy bearing, two outer race fault bearing is used. The healthy bearing, outer race fault type- 1 and outer race fault type- 2 bearing is shown in Figure 4, Figure 5 and Figure 6 respectively.

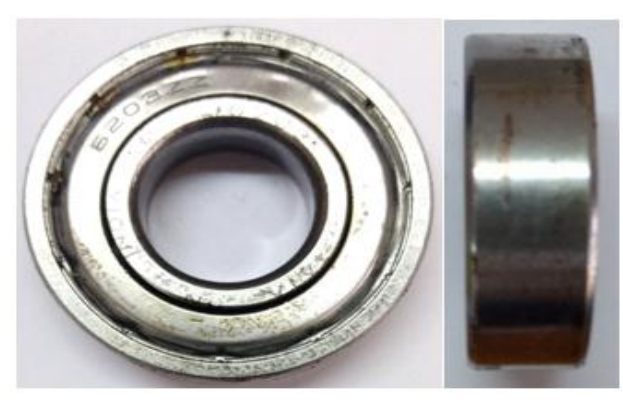

Figure 4. The healthy bearing

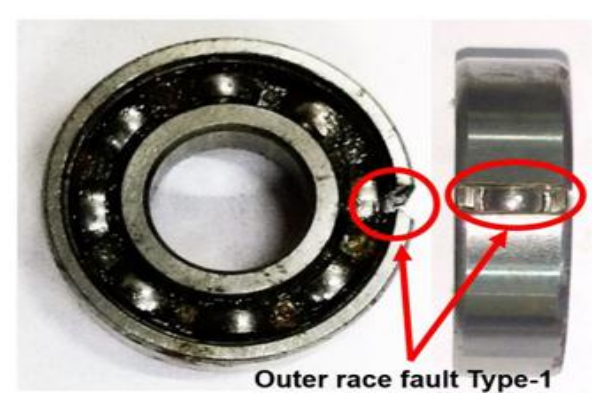

Figure 5. The type-1 defect bearing

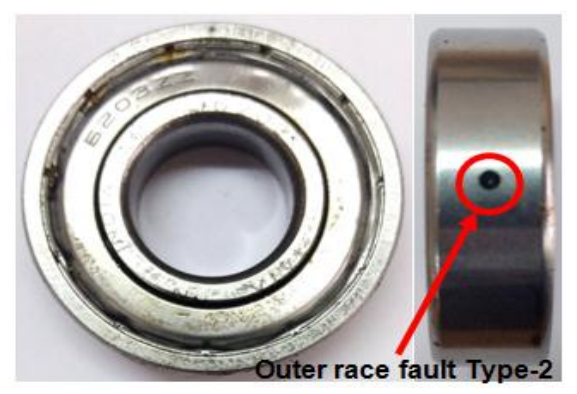

Figure 6. The type-2 bearing 
Initially the healthy bearing is mounted on the shaft and the corresponding acoustic signal is acquired. Similarly the three defective bearings are mounted one by one and the corresponding acoustic signal is acquired. All the acquired acoustic signals after filtration is processed for further analysis. The filtered acoustic signals are shown in Figure 7, Figure 8 and Figure 9.

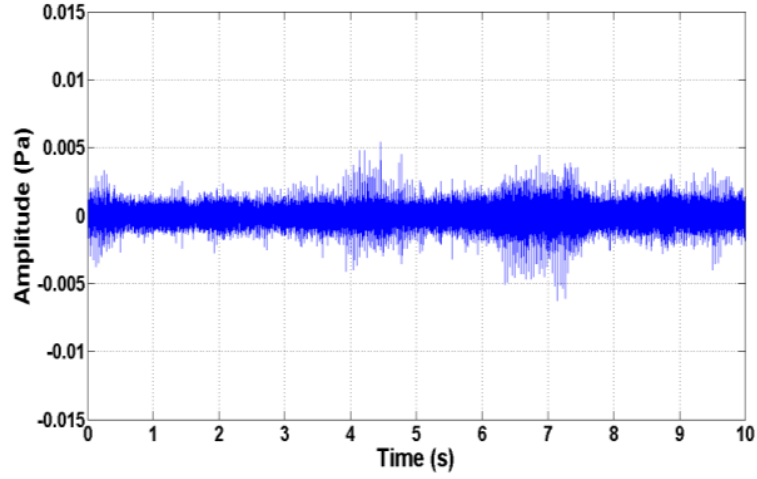

Figure 7. Filtered acoustic signal of healthy bearing (B1)

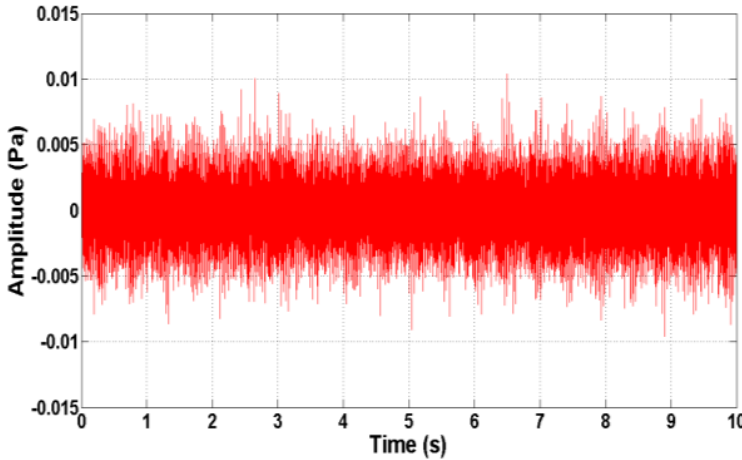

Figure 8. Filtered acoustic signal of outer race fault type-1 defect bearing (B2)

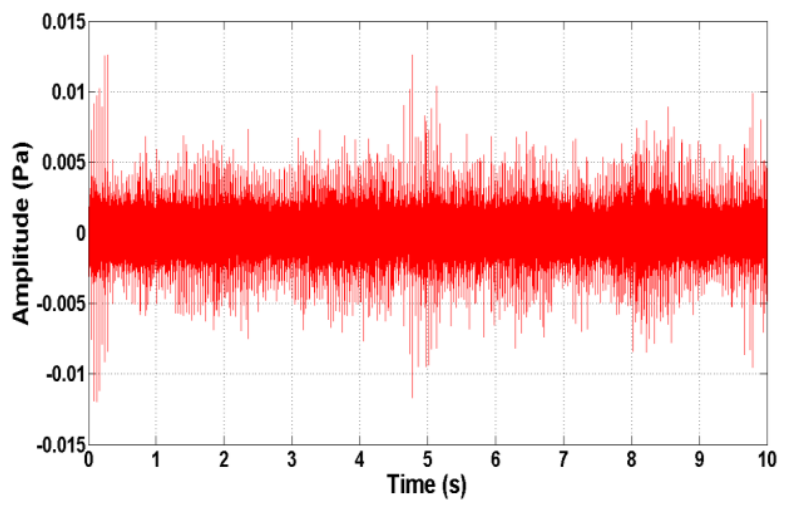

Figure 9. Filtered acoustic signal of outer race fault type-2 defect bearing (B3)

\section{RESULTS AND DISCUSSION}

This section shows the static (time), the frequency and the time-frequency (wavelet) analysis of the filtered acoustic signal to diagnose the fault in the bearings.

\subsection{Time domain (static) analysis}

Static parameters like, skewness, shape factor, kurtosis, RMS, crest factor, peak value etc. is calculated from the filtered acoustic signatures by using the mathematical formulas and is tabulated in Table 2. From the Table 2 the variations of the parameters for the faulty bearings as compared to healthy bearings are well observed. Also the difference in variations for different type of fault is well observed.

Table 2. Time Domain Parameter Comparison

\begin{tabular}{ccccc}
\hline S1. No & Static Parameters & Healthy Bearing & $\begin{array}{c}\text { Type-I } \\
\text { Defect Bearing }\end{array}$ & $\begin{array}{c}\text { Type-II } \\
\text { Defect Bearing }\end{array}$ \\
\hline 1 & Root mean square (RMS) & 0.0134 & 0.0261 & 0.0203 \\
2 & Mean & 0.4554 & 1.3406 & 0.9112 \\
3 & Peak value & 0.0773 & 0.1734 & 0.1432 \\
4 & Crest factor & 5.7737 & 6.6469 & 7.0564 \\
5 & Skewness & -0.0052 & -0.2564 & 0.1228 \\
6 & Kurtosis & 3.8532 & 9.0461 & 5.7542 \\
\hline
\end{tabular}




\begin{tabular}{ccccc}
\hline S1. No & Static Parameters & Healthy Bearing & $\begin{array}{c}\text { Type-I } \\
\text { Defect Bearing }\end{array}$ & $\begin{array}{c}\text { Type-II } \\
\text { Defect Bearing }\end{array}$ \\
\hline 7 & & & 0.1104 & 0.035 \\
8 & Variance & 0.0143 & 0.0261 & 0.0203 \\
9 & Standard deviation & 0.0134 & 665.9750 & 624.7892 \\
10 & Clearance factor & 716.0322 & 10.7450 & 9.4580 \\
11 & Impulse factor & 7.4381 & 1.6165 & 7.4381 \\
\hline
\end{tabular}

\subsection{Frequency domain (FFT) analysis}

Fast fourier transform (FFT) is used for this analysis. The FFT of healthy bearing and outer race fault type-1 defect bearing is compared and is shown in Figure 10. Similarly FFT comparison of the healthy bearing and the outer race fault type- 2 defect bearing is compared and is shown in Figure 11. Then the bearing characteristic frequency (BCF) also known as the outer race defect frequency (ORDF) is calculated using the geometric configuration of the bearing. Then the comparison between healthy and defective bearing at ORDF is shown in Figure 12 and Figure 13.

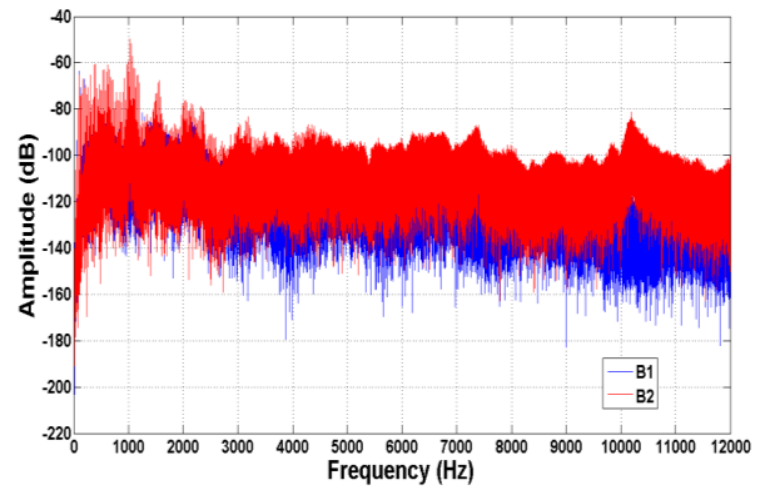

Figure 10. Comparison of FFT of healthy bearing (B1) and type- 1 defect bearing (B2)

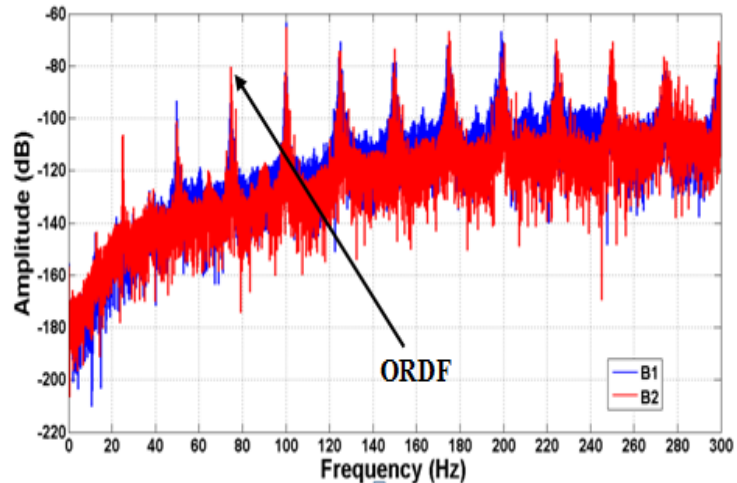

Figure 12. FFT comparison of healthy (B1) and type-1 defect bearing (B2) at BCF

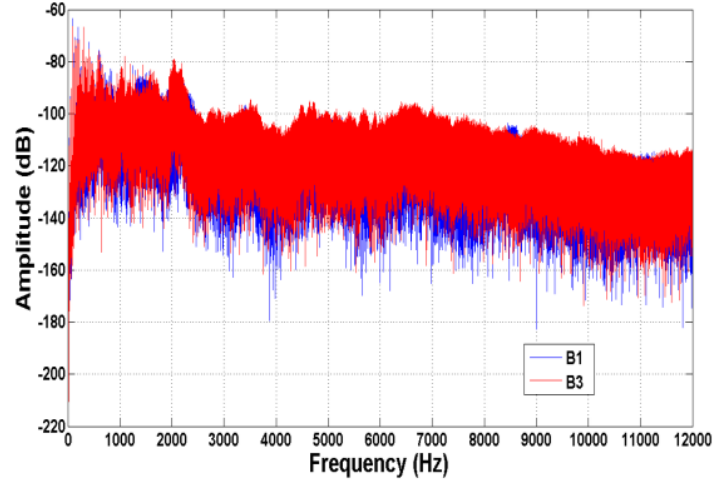

Figure 11. Comparison of FFT of healthy bearing (B1) and type-2 defect bearing (B3)

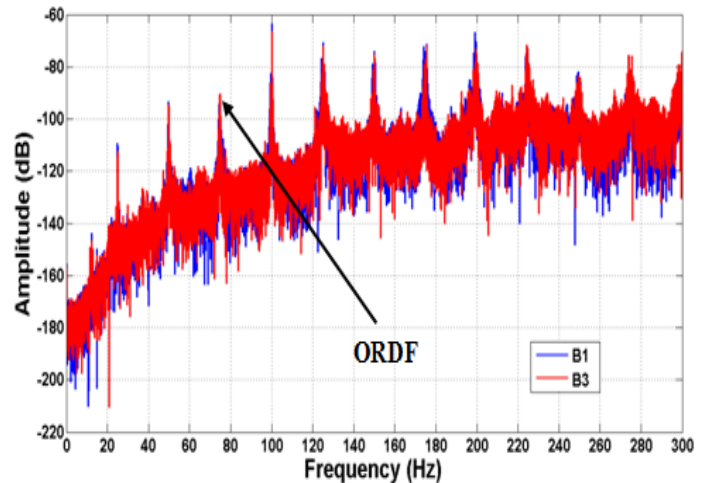

Figure 13. FFT comparison of healthy (B1) and type-2 defect bearing (B3) at BCF

\subsection{Time-frequency analysis}

Morlet wavelet is used for this purpose as the mother wavelet. 2D scalograms based on morlet wavelet are plotted for the acoustic signals. A difference is clearly visible around scale value of equivalent BCF for the healthy, type-1 defect and type-2 defect bearing as shown in Figure 14, Figure 15 and Figure 16 respectively. 


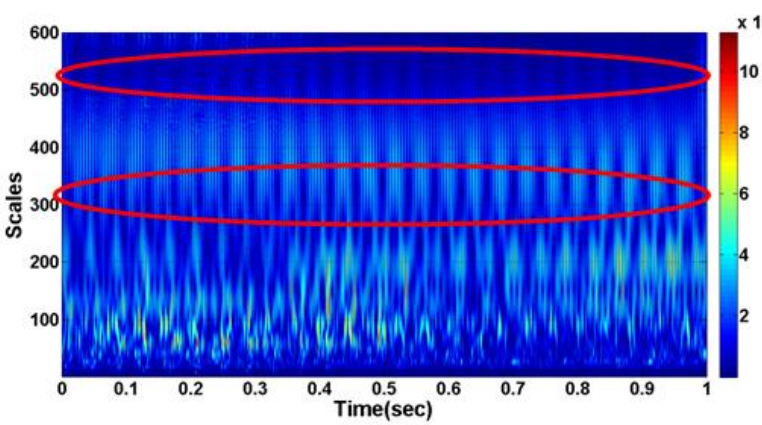

Figure 14. Scalogram for acoustic signature of healthy bearing (B1)

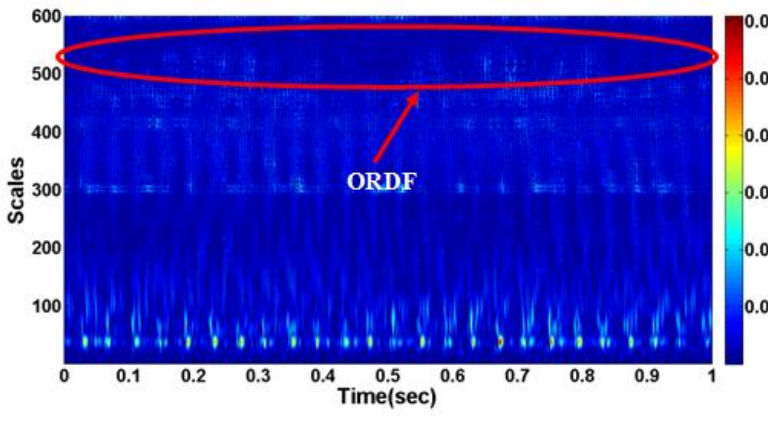

Figure 15. Scalogram for acoustic signature of outer race fault type-1 defect bearing (B2)

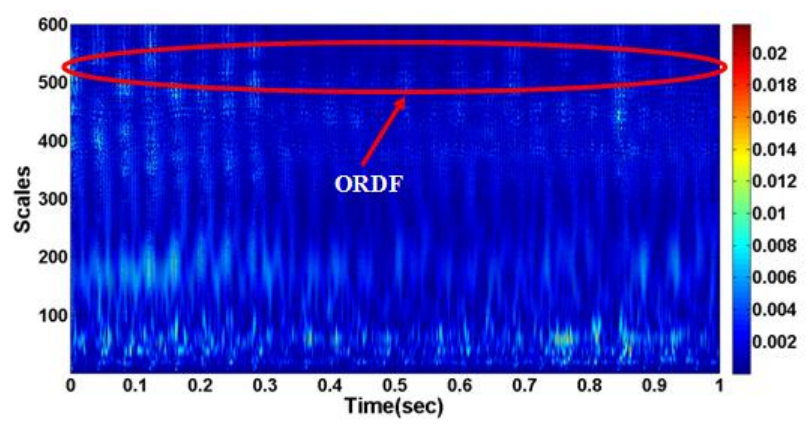

Figure 16 Scalogram for acoustic signature of outer race fault type-2 defect bearing (B3)

\section{CONCLUSION}

The present experimental work shows the application of active noise cancellation filtering technique to improve the signal to noise ratio of the acoustic signature before its use in analyzing the defects. The ANC filtering used in this work at the pre-processing stage is adaptive in nature and gives better performance in improving the SNR of the measured signal. The performance of the three different ANC techniques are compared and the best one is used at the pre-processing stage to remove the noise. In this work though static analysis gives the information about the defect present in the bearing but frequency analysis gives the comparison in a better way and the time-frequency analysis is more informative about the defect as it gives the information both in time and frequency scale. The scalograms from the wavelet analysis gives precise information of the defect present in the bearing. This work shows that the acoustic emission is a good alternative which can be used to diagnose the defect in the bearing of rolling element.

\section{REFERENCES}

[1] P. K. Kankar, S. C. Sharma, S. P. Harsha, "Fault Diagnosis of Ball Bearings using Continuous Wavelet Transform, Appl", Soft Comput, vol. 11, pp. 2300-2312, 2011.

[2] P. K. Kankar, Satish C. Sharma, S. P. Harsha, "Rolling Element Bearing Fault Diagnosis using Wavelet Transform”, Neurocomputing, vol. 74, pp. 1638-1645, May 2011.

[3] Wei Li, Mingquan Qiu, Zhencai Zhu, Bo Wu and Gongbo Zhou, "Bearing Fault Diagnosis Based on Spectrum Images of Vibration Signals”, Meas. Sci. Technol, vol. 27, 035005 (10pp), 2016

[4] M. Misiti, Y. Misiti, G. Oppenheim, J. M. Poggi, "Wavelets and Their Applications", 3rd ed., France: Hermes Science, 2003.

[5] Z. K. Peng, F. L. Chu, "Application of the Wavelet Transform in Machine Condition Monitoring and Fault Diagnostics: A Review With Bibliography”, Mechanical Systems and Signal Processing, vol. 18, pp. 199-221, Mar 2004.

[6] J. I. Taylor, "The Vibration Analysis Hand Book", 2nd ed., USA: Vibration Consultants, 2003.

[7] V. Dhanush Abhijit, V. Sugumaran and K. I. Ramachandran, "Fault Diagnosis of Bearings Using Vibration Signals and Wavelets", Indian Journal of Science and Technology, vol. 9, 2016. 
[8] P. Shakya, A. K. Darpe and M. S. Kulkarni, "Vibration-based Fault Diagnosis in Rolling Element Bearings: Ranking of Various Time, Frequency and Time-frequency Domain Data-based Damage Identification Parameters", The International Journal of Condition Monitoring, vol. 3, pp. 53-62, Oct 2013.

[9] B. Li, P. Zhang, Z. Wang, S. Mi, Y. Zhang, "Gear Fault Detection Using Multi-scale Morphological Filters", Measurement, vol. 44, pp. 2078-2089, Dec 2011.

[10] A. Albarbar, F. Gu, A.D. Ball, A. Starr, "Acoustic Monitoring of Engine Fuel Injection Based on Adaptive Filtering Techniques", Appl. Acoust, vol. 70, pp. 247-255, 2009.

[11] T. G. Manjunath, Ashok Kusagu, "Fault Diagnosis and Reconfiguration of Multilevel Inverter Switch Failure a Performance Perspective", International Journal of Electrical and Computer Engineering, vol.6, pp. 2610-2620, Dec 2016.

[12] M. A. Talib, N. A. Muhamad, Z. A. Malek, B. T. Phung, "Fault Identification of In Service Power Transformer Using Depolarization Current Analysis", International Journal of Electrical and Computer Engineering, vol. 7, pp. 559-567, Apr 2017.

[13] C. W. Liao, J. Y. Lin, "New FIR Filter-based Adaptive Algorithms Incorporating with Commutation Error to Improve Active Noise Control Performance", Automatica, vol. 43, pp. 325-331, 2007.

[14] M. I. Troparevsky, C. E. D'Attellis, "On the Convergence of the LMS Algorithm in Adaptive Filtering”, Signal Process, vol. 84, pp. 1985-1988, 2004.

[15] Shubhra Dixit, Deepak Nagaria, "LMS Adaptive Filters for Noise Cancellation", International Journal of Electrical and Computer Engineering, vol. 7, pp. 2520-2529, Oct 2017.

\section{BIOGRAPHIES OF AUTHORS}

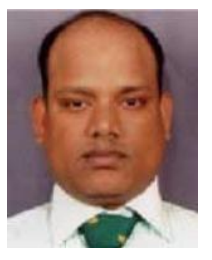

Sudarsan Sahoo was born on July 1980 in Cuttack, India. He received his Bachelor degree from SLIET, Punjab, India in Instrumentation Engineering and Master degree from Indian Institute of Science, Bangalore, India in Instrumentation engineering. He is currently Assistant Professor at the Department of Electronics and Instrumentation Engineering, National Institute of Technology Silchar (NIT Silchar), India. His research is specialized in acoustic and biomedical signal processing, intelligent instrumentation and industrial noise control.

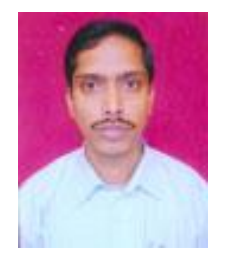

Jitendra Kumar Das was born in 1970 in cuttack, India. He received both his Master degree and $\mathrm{PhD}$ degree from National Institute of Technology Rourkela (NIT Rourkela),India. He is currently working as Professor at School of Electronics Engineering, KIIT University, India. His research is specialized in VLSI, Signal processing. 